\title{
CARACTERIZAÇÃO DAS PROPRIEDADES FÍSICO- QUÍMICAS, TECNOLÓGICAS E VISCOAMILOGRÁFICAS DA FARINHA DE PUPUNHA (BACTRIS GASIPAE KUNT).
}

\author{
M. B. PIRES ${ }^{1}$, E. R. AMANTE ${ }^{2}$, A. M. C. RODRIGUES ${ }^{1}$, L. H. M. da SILVA ${ }^{1}$. \\ ${ }^{1}$ Universidade Federal do Pará, Programa de Pós Graduação em Ciência e Tecnologia de \\ Alimentos \\ ${ }^{2}$ Universidade Federal de Santa Catarina , Programa de Pós Graduação em Ciência dos \\ Alimentos. \\ E-mail para contato: marliapires@ufpa.br
}

\begin{abstract}
RESUMO - Visando sua utilização na elaboração de produtos alimentícios, foram obtidas diferentes farinhas de pupunha (Bactris gasipaes Kunth) da variedade Vaupés. Avaliou-se a composição físico-química, as propriedades tecnológicas (viscosidade, capacidade de absorção de água, absorção de óleo, capacidade emulsificante e capacidade de formação de espuma) e a morfologia das farinhas de pupunha. Os resultados apresentaram diferença de cor entre elas, proporcionada pelo tratamento térmico e presença ou ausência de casca, a determinação da densidade demonstrou não haver diferença significativa entre os lotes. Os valores de umidade ficaram de acordo com a legislação vigente, que preconiza máximo de $15 \%$ de umidade. Foram encontrados teores consideráveis de lipídeos $(\geq 10 \%)$ e carboidrato $(\geq 59 \%)$. Os diferentes lotes apresentaram-se como excelente fonte energética ( $\geq 390 \mathrm{kcal} / 100 \mathrm{~g}$ ). O ácido graxo predominante nas farinhas de pupunha foi o ácido oleico (C18: 1), que representou em média $45 \%$ de sua composição. Os perfis viscoamilográficos determinados foram bastante diferentes, evidenciando, uma redução dos valores de viscosidade nos lotes que passaram por tratamento térmico. As micrografias eletrônicas apresentam a estrutura do amido com caraterísticas arredondadas e tamanhos não homogêneos.
\end{abstract}

\section{INTRODUÇÃO}

Segundo Kerr et al. (1997) o fruto, conhecido no Brasil como pupunha, é bastante utilizado na alimentação, sendo rico em proteínas, carboidratos, fibras, caroteno (pró-vitamina A), ácido ascórbico, minerais, principalmente ferro, cálcio e fósforo. Os frutos são consumidos cozidos e prestam-se à extração de óleo ou à produção de farinhas para as mais variadas finalidades (GOIA et al., 1997). 
Uma das formas de industrialização deste fruto é na forma de farinha, em estudos realizados no Brasil e na Costa Rica demonstrou o uso de farinha de pupunha em panificação e pastelaria, em substituição à farinha de trigo e milho, com resultados satisfatórios (CLEMENT e MORA URPI, 1988). O incentivo quanto ao seu consumo poderiam solucionar problemas de saúde pública, uma vez que já foi comprovada a eficiência da utilização da pupunha como fonte de vitamina A (YUYAMA e COZZOLINO, 1996).

Com interesse de atender a indústria de alimentos, que vem enfrentando um mercado cada vez mais competitivo, consumidores que procuram por produtos saudáveis e com característica sensoriais agradáveis, agregando valor aos mesmos utilizando-se para isso de desenvolvimento tecnológico se propôs a elaboração e caracterização físico-química e das propriedades tecnológicas da farinha de pupunha (Bactris gasipaes Kunth), variedade Vaupés, visando sua utilização na elaboração de produtos alimentícios.

\section{MATERIAIS E MÉTODOS}

\subsection{Matéria prima}

Os frutos de pupunha (Bactris gasipaes Kunth) da variedade Vaupés foram adquiridos no mercado do Ver-o-peso, em Belém no estado do Pará.

\subsection{Obtenção da farinha}

Os frutos foram separados em lotes (Lote 1: Pupunha sem cozimento sem casca; Lote 2: Pupunha sem cozimento com casca; Lote 3: Pupunha com cozimento sem casca e Lote 4: Pupunha com cozimento com casca), o cozimento foi em autoclave a temperatura de $121^{\circ} \mathrm{C}$ por 60 minutos, os frutos pertencentes aos diferentes lotes foram secos em secador de leito fixo, sob temperatura de $55^{\circ} \mathrm{C}$ por em média 42 horas. Após a secagem os frutos foram triturados em moinho de martelos contínuo, seguida de moinho de facas contínuo e embaladas a vácuo e armazenadas em temperatura ambiente, até o momento das análises.

\subsection{Caracterização físico-química}

A análise de cor foi realizada em colorímetro, operando no sistema CIE ( $\left.L^{*}, a^{*}, b^{*}\right)$, da marca HUNTER modelo COLOR QUEST XE, sendo as coordenadas de cromaticidade: L* para luminosidade, $a^{*}$ para intensidade da cor vermelho e $b^{*}$ para intensidade da cor amarela. As densidades absolutas das partículas de farinha foram determinadas pelo método do picnômetro, por deslocamento do xileno a $30^{\circ} \mathrm{C}$, segundo Schoch e Leach (1964).

A composição centesimal (umidade, proteínas, lipídeos, cinzas e carboidratos), $\mathrm{pH}$ e acidez foram determinados segundo métodos descritos pela AOAC (2002). O valor calórico foi calculado com base na composição centesimal, utilizando os coeficientes de Atwater 
(WATT; MERRILL, 1963), que considera 4kcal/g para proteínas e carboidratos e 9kcal/g para lipídeos.

A extração dos ácidos graxos foi feita usando bligh e dyer, e a composição desses ácidos graxos foi determinada pela conversão de ácidos graxos em ésteres metílicos (FAMEs), com base no método proposto por Rodrigues et al. (2010) e detectados utilizando cromatografia gasosa. A extração de carotenos presentes nas farinhas, foi feita segundo o método de Rodriguez -Amaya, (1994).

\subsection{Caracterização morfológica da farinha}

As amostras de farinha de pupunha foram submetidas a microscopia eletrônica de varredura (MEV) em microscópio eletrônico de varredura modelo ZEISS DSM 940 A. Para a microscopia óptica, as amostras foram visualizadas em microscópio óptico com luz polarizada Olympus SZH10, acoplado a uma câmera fotográfica Olympus.

\subsection{Propriedades viscoamilográficas}

A determinação das propriedades de pasta foi feita em equipamento Rapid Visco Analyser (RVA), da marca PERTEN, série RVA 4500. Cujos parâmetros estão de acordo com AACC número 76-21 (AACC, 2000).

\subsection{Análise estatística}

Todas as análises foram conduzidas em triplicata e os dados expressos como média aritmética e desvio padrão (DP). Os dados foram submetidos à análise de variância (ANOVA), teste de Tukey á nível de $5 \%$ de significância, para comparação das médias.

\section{RESULTADOS E DISCUSSÕES}

\subsection{Caracterização físico-química das farinhas de pupunha}

Os resultados encontrados para determinação de cor e densidade das farinhas de pupunha encontram-se expressos na Tabela 1, onde se observa que a farinha de pupunha sem cozimento e sem casca (FP1) apresenta intensidade menor de vermelho e amarelo. Pode-se observar que a coloração amarela e o vermelho elevaram-se após o tratamento térmico, isto se deve ao fato de alguns carotenoides modificarem sua estrutura após o cozimento. As moléculas dos carotenoides, dependendo da conformação, possuem um sistema de duplas ligações que constituem o grupo cromófago responsável pela cor que proporciona aos alimentos (RIBEIRO e SERAVALHI, 2004).

Tabela 1- Determinação de cor nas farinhas de pupunha

\begin{tabular}{lll}
\hline Amostra & Cor & Densidade \\
\hline
\end{tabular}




\begin{tabular}{ccccc}
\hline & $\mathrm{L}^{*}$ & $\mathrm{a}^{*}$ & $\mathrm{~b}^{*}$ & Densidade $\left(\mathrm{g} \mathrm{cm}^{-3}\right)$ \\
\hline FP1 & $81,13 \pm 0,4^{\mathrm{a}}$ & $2,69 \pm 0,1^{\mathrm{b}}$ & $18,45 \pm 0,1^{\mathrm{c}}$ & $1,606 \pm 0,2^{\mathrm{a}}$ \\
FP2 & $78,17 \pm 0,3^{\mathrm{c}}$ & $3,75 \pm 0,3^{\mathrm{a}}$ & $21,1 \pm 0,4^{\mathrm{b}}$ & $1,673 \pm 0,2^{\mathrm{a}}$ \\
FP3 & $82,32 \pm 0,07^{\mathrm{b}}$ & $3,83 \pm 0,2^{\mathrm{a}}$ & $26,03 \pm 0,5^{\mathrm{a}}$ & $1,301 \pm 0,1^{\mathrm{a}}$ \\
FP4 & $76,21 \pm 0,4^{\mathrm{d}}$ & $3,77 \pm 0,1^{\mathrm{a}}$ & $25,06 \pm 0,8^{\mathrm{a}}$ & $1,320 \pm 0,3^{\mathrm{a}}$ \\
\hline
\end{tabular}

FP1: sem cozimento sem casca; FP2: sem cozimento com casca; FP3: com cozimento sem casca e FP4: com cozimento com casca. Valores médios de três repetições com seus desvios padrões. Letras diferentes entre linhas representam diferença significativa a 95\% de significância.

A umidade é um parâmetro importante a ser avaliado, pois indica se a secagem foi adequada. Segundo a Resolução CNNPA N 12 da ANVISA (BRASIL, 1978), que regulamenta os parâmetros de qualidade da farinha de trigo, a umidade deve estar abaixo de 15\%. Logo, como pode se observar na Tabela 2, com exceção da farinha sem cozimento com casca, todas as outras estão de acordo essa legislação. Os teores de lipídeos e proteínas estão de acordo com Goia et al. (1993) (8,9- 22,4 g/100g b.s. e 4,1- 6,6 g/100g b.s, respectivamente). As farinhas podem ser consideradas excelente fonte de carboidratos, concentração maior do que $59 \%$ e excelente fonte de calorias.

Tabela 2- Composição das farinhas de pupunha (Bactris gasipaes Kunth)

\begin{tabular}{ccccc}
\hline Componente & FP1 & FP2 & FP3 & FP4 \\
\hline Umidade (g/100g) & $9,45 \pm 0,1^{\mathrm{a}}$ & $17,64 \pm 0,2^{\mathrm{b}}$ & $9,47 \pm 0,06^{\mathrm{a}}$ & $13,44 \pm 0,1^{\mathrm{c}}$ \\
Lipídeos (g/100g) & $10,09 \pm 0,6^{\mathrm{d}}$ & $13,53 \pm 0,4^{\mathrm{c}}$ & $15,27 \pm 0,5^{\mathrm{b}}$ & $20,42 \pm 0,3^{\mathrm{a}}$ \\
Cinzas (g/100g) & $1,42 \pm 0,03^{\mathrm{c}}$ & $1,52 \pm 0,01^{\mathrm{a}, \mathrm{b}}$ & $1,48 \pm 0,02^{\mathrm{b}}$ & $1,56 \pm 0,01^{\mathrm{a}}$ \\
Proteínas (g/100g) & $4,83 \pm 0,06^{\mathrm{c}}$ & $5,09 \pm 0,02^{\mathrm{a}}$ & $4,68 \pm 0,1^{\mathrm{c}}$ & $5,00 \pm 0,01^{\mathrm{b}}$ \\
Carboidratos (g/100g) & $74,19 \pm 0,5^{\mathrm{a}}$ & $62,19 \pm 0,6^{\mathrm{c}}$ & $69,09 \pm 0,4^{\mathrm{b}}$ & $59,56 \pm 0,3^{\mathrm{d}}$ \\
pH & $3,35 \pm 0,07^{\mathrm{c}}$ & $3,9 \pm 0,0^{\mathrm{a}}$ & $3,2 \pm 0,07^{\mathrm{d}}$ & $3,6 \pm 0,14^{\mathrm{b}}$ \\
$\begin{array}{c}\text { Acidez titulável } \\
\text { Carotenos totais } \\
\text { (mg/100g) }\end{array}$ & $3,26 \pm 0,2^{\mathrm{a}}$ & $2,44 \pm 0,1^{\mathrm{b}}$ & $2,40 \pm 0,1^{\mathrm{b}}$ & $1,69 \pm 0,02^{\mathrm{b}}$ \\
Valor calórico & $6,33 \pm 0,02^{\mathrm{b}}$ & $15,67 \pm 0,1^{\mathrm{d}}$ & $8,71 \pm 0,04^{\mathrm{a}}$ & $16,34 \pm 0,08^{\mathrm{c}}$ \\
$\quad(\mathrm{kcal} / 100 \mathrm{~g})$ & $406,606 \pm 0,6^{\mathrm{c}}$ & $390,89 \pm 0,8^{\mathrm{d}}$ & $432,51 \pm 0,8^{\mathrm{b}}$ & $442,02 \pm 0,2^{\mathrm{a}}$ \\
\hline
\end{tabular}

FP1: sem cozimento sem casca; FP2: sem cozimento com casca; FP3: com cozimento sem casca e FP4: com cozimento com casca. Valores médios de três repetições com seus desvios padrões. Letras diferentes entre colunas representam diferença significativa a $95 \%$.

Os valores apresentados na Tabela 2 mostram o efeito do tratamento térmico sobre os carotenoides totais para os lotes de farinhas estudados. As farinhas que foram submetidas a tratamento térmico apresentam maiores teores de carotenoides, segundo Garbanzo (2011) isso se deve ao fato de o aquecimento alterar a estrutura dos carotenoides, alterando sua conformação (cis, trans). 
Após determinação verificou-se que o ácido graxo predominante nas farinhas de pupunha foi o ácido oleico (C18:1), que representou em média 45\% de sua composição, seguido do ácido Palmítico (C16:0), com 30\% dos ácidos que constituem as farinhas de pupunha. Os resultados encontrados para os ácidos graxos das farinhas de pupunha estão de acordo com Ciprona (1986), Rojas et al. (1994) e Yuyama et al. (2003). Os ácidos graxos, através da alimentação, são utilizados como fonte de energia para o funcionamento do organismo. Aproximadamente $40 \%$ do total da necessidade diária de ácidos graxos são obtidos através da dieta (ROJAS, 1994).

\subsection{Propriedades viscoamilograficas}

Na Tabela 4 são apresentados os principais pontos do viscoamilograma para as quatro farinhas de pupunha.

Tabela 4- Propriedades de pasta das farinhas de pupunha

\begin{tabular}{ccccccc}
\hline Amostras & $\begin{array}{c}\text { Tp } \\
\left({ }^{\circ} \mathrm{C}\right)\end{array}$ & $\begin{array}{c}\text { VM } 95^{\circ} \mathrm{C} \\
(\mathrm{Cp})\end{array}$ & $\begin{array}{c}\text { VF 50 } \\
(\mathrm{Cp})\end{array}$ & $\begin{array}{c}\text { Quebra } \\
(\mathrm{Cp})\end{array}$ & $\begin{array}{c}\text { Retrogradação } \\
(\mathrm{Cp})\end{array}$ & $\begin{array}{c}\text { Tp } \\
(\mathrm{min})\end{array}$ \\
\hline FP1 & $93,15 \pm 2^{\mathrm{a}}$ & $461,67 \pm 1^{\mathrm{b}}$ & $458,66 \pm 3^{\mathrm{b}}$ & $367,33 \pm 4^{\mathrm{b}}$ & $364,33 \pm 3^{\mathrm{a}}$ & $11,69 \pm 2^{\mathrm{a}}$ \\
FP2 & $94,9 \pm 0,2^{\mathrm{a}}$ & $672,67 \pm 1^{\mathrm{a}}$ & $649,67 \pm 3^{\mathrm{a}}$ & $590 \pm 5^{\mathrm{a}}$ & $547 \pm 4^{\mathrm{b}}$ & $12,22 \pm 0,4^{\mathrm{a}}$ \\
FP3 & $62 \pm 2^{\mathrm{b}}$ & $33 \pm 1^{\mathrm{c}}$ & $29,67 \pm 0,5^{\mathrm{c}}$ & $33 \pm 1^{\mathrm{d}}$ & $29,66 \pm 0,5^{\mathrm{d}}$ & $11,310,2^{\mathrm{a}}$ \\
FP4 & $52,67 \pm 3^{\mathrm{c}}$ & $38,33 \pm 7^{\mathrm{c}}$ & $30,33 \pm 2^{\mathrm{c}}$ & $75,67 \pm 3^{\mathrm{c}}$ & $67,67 \pm 8 \mathrm{c}$ & $10,93 \pm 0,4^{\mathrm{a}}$ \\
\hline
\end{tabular}

FP1: sem cozimento sem casca; FP2: sem cozimento com casca; FP3: com cozimento sem casca e FP4: com cozimento com casca. Média de três repetições com seus desvios padrões. Letras diferentes entre linhas representam diferença significativa a 95\%. Tp: Temperatura inicial de pasta; VM: Viscosidade máxima; VF: Viscosidade final; tp: Tempo de pico. cP: centipoise.

Os perfis de viscosidade das farinhas diferenciaram, pois houve efeito do tipo de tratamento (sem cozimento e com cozimento) e a composição das mesmas (sem casca e com casca). Os parâmetros temperatura de pasta, viscosidade máxima e viscosidade final apresentaram valores menores para a farinha com cozimento em relação a sem cozimento, isto deve-se ao fato de o tratamento térmico ter pré-gelatinizado o amido presente nestas farinhas. Considerando as temperaturas de empastamento dos diferentes lotes de farinha, pode-se dizer que do ponto de vista energético as farinhas cozidas são mais interessantes, já que produzem pastas viscosas mais rapidamente. Já a elevação da temperatura de pasta, para as farinhas sem cozimento, pode ser associada a uma redução da região cristalina do grânulo, gerando aumento de viscosidade e de tendência a retrogradação (ADEBOWALE et al., 2005).

\subsection{Caracterização morfológica das farinhas}

A Figura 2 apresenta as micrografías das farinhas de pupunha, já a Figura 3, mostra a organização externa dos grânulos de amido que constituem as farinhas de pupunha, com a visualização da cruz de malta. 

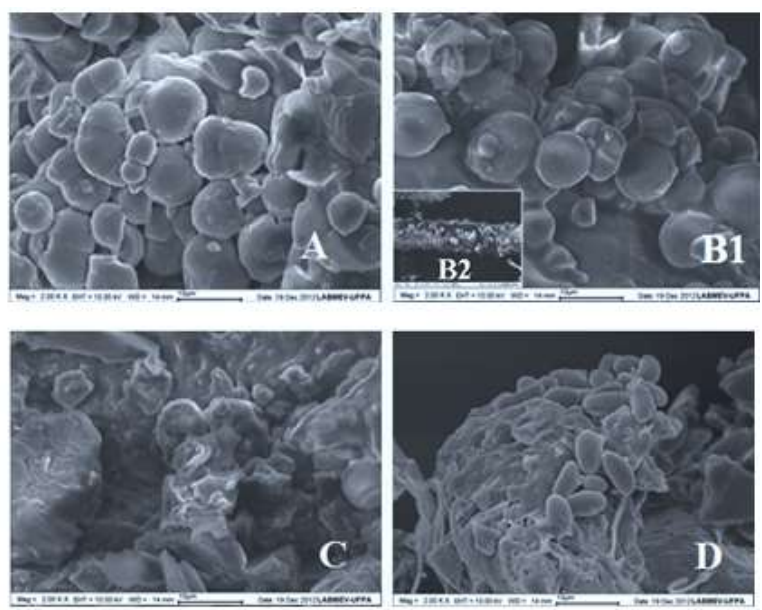

Figura 2- Microscopia eletrônica de varredura das farinhas de pupunha (aumento de 2000x). A- Farinha de pupunha sem cozimento sem casca; B- Farinha de pupunha sem cozimento com casca; C- Farinha de pupunha com cozimento sem casca e D- Farinha de pupunha com cozimento com casca.

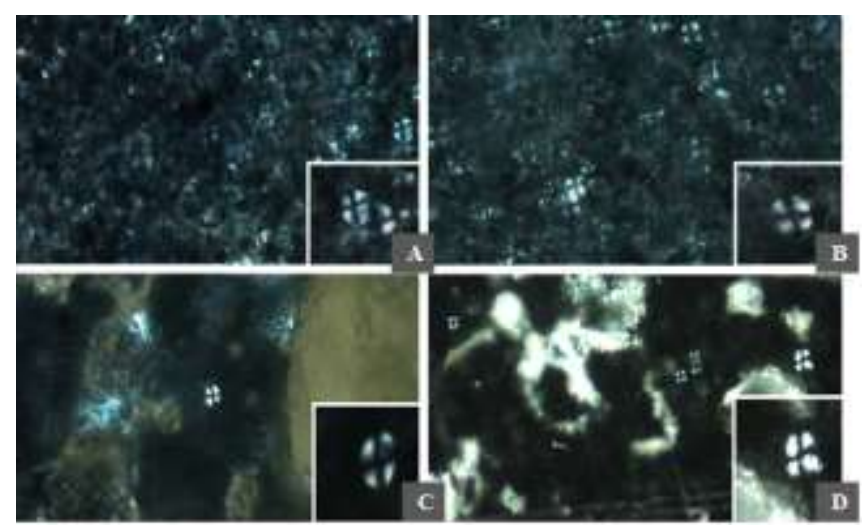

Figura 3- Imagens de microscopia óptica sob luz polarizada das diferentes farinhas de pupunha. A- sem cozimento sem casca; B - sem cozimento com casca; C- com cozimento sem casca e D- com cozimento com casca.

As micrografias ilustram a morfologia das farinhas de pupunha, onde se pode observar claramente na Figura 2 A e B1 a estrutura do amido com caraterística arredondada e tamanho não homogêneo. Na Figura 2- B2 pode-se observar a presença da estrutura vegetal referente as fibras oriundas da casca. As Figuras 2C e D mostram claramente que após o processo de cozimento houve gelatinização do amido e das fibras presentes nas farinhas. Quando se avalia as microscopias ópticas se visualiza perfeitamente a cruz de malta, observa-se que após o processo de cozimento estes grânulos alteraram sua estrutura interna, mas ainda pode ser observado, o que sugere resistência deste grânulo de amido a temperatura de processo ao qual foram submetidas (GARCIA et al., 1996). 


\section{CONCLUSÕES}

Os resultados obtidos após a avaliação da composição das farinhas estão de acordo com estudos realizados por outros autores, demonstrando o sucesso do processo de obtenção de farinha de pupunha a partir da variedade Vaupés. A farinha de pupunha pode ser considerada excelente fonte de carboidratos e excelente fonte de energia. A inserção da casca de pupunha na farinha eleva o conteúdo principalmente de lipídeos, carotenoides e ácidos graxos, agregando valor nutricional à farinha de pupunha. As farinhas apresentaram comportamentos diferentes de viscosidade, principalmente relacionado com o cozimento dos frutos.

\section{REFERENCIAS}

AACC. American Association of Cereal Chemists). Approved Methods of the AACC. St. Paul, AACC, p76-21, 2000.

ADEBOWALE, K. O.; AFOLABI, T. A.; OLU - OWOLABI, B.I., Hydrothermal trestementes of finger millet (Eleucine Corona) start. Food Hydrocol. Wresch., v. 19, p. 974983, 2005.

AOAC; Official Methods of Analysis of Association of Official Analytical Chemists. 17th ed. Washington, 2002.

BRASIL, Resolução CNNPA No 12, de 24/ 07/ 1978. Regulamento técnico sobre padrões de identidade e qualidade da farinha de trigo. Diário oficial. Brasília, DF.

CIPRONA. Usos industriales del pejibaye (Bactris gasipaes). Informe de Investigación del Centro de Investigaciones de Productos Naturales (CIPRONA), Universidad de Costa Rica, San José, Costa Rica, 1986.

CLEMENT, C.R.; MORA URPÍ, J.. Phenotypic variation of peach palm observed in the Amazon basin. In: Clement, C.R.; Coradin, L. (eds.). Final report (revised): Peach palm (Bactris gasipaes H.B.K.) germplasm bank. U.S. Agency for International Development (grant number DAN-5542-G-SS-2093-00), Instituto Nacional de Pesquisas da Amazônia, Centro Nacional de Recursos Genéticos/Embrapa, Manaus, AM. 1988 pp. 2005, 20-54.

GARBANZO, C. R; PÉREZ, A. M.; CARMONA, J. B.; Identification and quantification of carotenoids by HPLC-DAD during the process of peach palm (Bactris gasipaes H.B.K.) flour, Journ. of Food Researc. Internat., São José, Costa Rica, 2011.

GARCIA, V.; COLONNA, P.; LOURDIN, D.; BULEON, A.; BIZOT, H.; OLLIVON, M. Thermal transitions of cassava at intermediate water contents, Journ. of Therm. Analys., v. 47, p. 1213-1228. 1996. 
GOIA, C. H.; ANDRADE, J. S.; ARAGÃO, C. G. Composição química da farinha de pupunha. Revist. Quími. Indust., v. 2, n. 2, p. 48-54, 1993.

KERR, L.S.; CLEMENT, R.N.S.; CLEMENT, C.R.; KERR, W.E. Cozinhando com a Pupunha. Instituto Nacional de Pesquisas da Amazônia, Manaus, AM. 95 p. 1997.

RIBEIRO, E. P; SERAVALLI. A.G. Química de alimentos. Instituto Mauá de tecnologia. Editora Edgar. Blucher Ltda, 1 edição, São Paulo, p. 155- 157, 2004.

RODRIGUES, A.M.C.; DARNET, S.; SILVA, L.H.M. Fatty Acid Profiles and Tocopherol Contents of Buriti (Mauritia flexuosa), Patawa (Oenocarpus bataua), Tucuma (Astrocaryum vulgare), Mari (Poraqueiba paraensis) and Inaja (Maximiliana maripa) Fruits. Journ. Brazil. Chem. Societ.. v.21, No. 10, 2000-2004, 2010.

RODRIGUES-AMAYA, D. A Guide to Carotenoid Analysis in Foods. ILSI Press, Washington, 1999, p 37-5.

ROJAS, J. M. Chemometric classificats of two peach palm (Bactri gasipaes H.B.K.). Londraces (Juruion and vaupes) Perú, 1994.

WATT, B; MERRILL, AL. Composition of food: Raw, Processed, Prepared. Washington, D. C: Consumer end food economics research division/ Agricult. Research. Service., 1963.

YUYAMA, K. O.; COZZOLINO, S. M. F. Efeito da suplementação com pupunha como fonte de vitamina A em dieta: estudo em ratos. Revist. de Saúd. Públic., v. 30, n. 1, 1996.

YUYAMA, L. K.; AGUIAR, J. P.; YUYAMA, K.; CLEMENT, C. R.; MACEDO, S. H.; FAVARO, D. I.; AFONSO, C.; VASCONCELLOS, M. B.; PIMENTEL, S. A.; BADOLATO, E. S.; VANNUCCHI, H. Chemical composition of the fruit mesocarp of three peach palm (Bactris gasipaes) populations grown in central Amazonia, Brazil. Int. Journ. Food Sci. Nutr., v. 54, n. 1, 2003, p. 49-56, 